\title{
The treatment of trigeminal neuralgia by posterior fossa microsurgery
}

\author{
CBT ADAMS, ANDREW H KAYE, PJ TEDDY \\ From the Department of Neurological Surgery, The Radcliffe Infirmary, Oxford, UK
}

SUMMARY Between 1972 and 198157 patients underwent posterior fossa exploration in Oxford by a single surgeon for the treatment of trigeminal neuralgia. Fifty-four of these had either partial or total section of the trigeminal sensory root, 2 had microvascular decompression operations and one had both a partial sensory root section and microvascular decompression. There was no mortality and no significant morbidity. Fifty-four patients were followed up for a mean period of 4.5 years. Fifty-two patients $(96 \%)$ had either no further pain or only minor twinges requiring no further treatment. Two other patients who had partial sections suffered no further trigeminal neuralgia after subsequent total sections. One patient who had a partial root section developed anaesthesia dolorosa and one who had a microvascular decompression developed painful dysaesthesia. All patients having partial root sections retained previously intact corneal responses-the sensory impairment in the face corresponded to the appropriate part of the portio major cut. Only $11 \%$ of patients were found to have a vascular abnormality.

Although most patients suffering from tic douloureux are able to obtain some control of their pain with carbamazepine the relief is often temporary and surgical treatment is eventually required. Numerous surgical procedures on either the central or more peripheral portion of the trigeminal nerve have been used. The preganglionic sensory root section was pioneered by Horsley ${ }^{1}$ and Frazier $^{23}$ through a middle cranial fossa approach. Dandy ${ }^{4}$ sectioned the sensory root of the trigeminal nerve in 250 cases via the posterior fossa. Operating without a microscope he achieved pain relief in over $90 \%$ of patients and observed that approximately $30 \%$ of these cases showed gross lesions (arterial, venous or neoplastic) compressing the nerve root. Jannetta ${ }^{5}$ using microneurosurgical techniques considers that vascular compression of the sensory root is the cause in approximately $90 \%$ of his cases. This observation has been confirmed by other workers ${ }^{6-10}$ and has consequently led to the formulation of a theory of vascular compression as the principle cause not only of trigeminal neuralgia but also of hemifacial spasm.

Address for reprint requests: CBT Adams, Department of Neurological Surgery, The Radcliffe Infirmary, Oxford, OX2 6HE, UK.

Received 18 March 1982 and in revised form 31 July 1982 Accepted 20 August 1982
However, we have not found this high incidence of vascular compression in cases of hemifacial spasm ${ }^{11}$ ${ }^{12}$ or in this series of patients with trigeminal neuralgia treated by posterior fossa surgery.

We report our experience of the treatment of 57 patients by posterior fossa surgery, including 55 who have been treated with section of either whole or part of the sensory division of the 5th cranial nerve.

\section{Patients and methods}

Since 197255 patients with trigeminal neuralgia have been treated by one surgeon (CBTA) at the Department of Neurological Surgery, The Radcliffe Infirmary, Oxford with section of either part or whole of the sensory division of the 5th cranial nerve via a posterior fossa approach. Two additional patients were treated by microvascular decompression of the sensory division using Ivalon sponge. At the time of operation the ages of the patients ranged from 31 to 83 years with a mean of 59 years, 11 patients being over 70 years. The length of symptoms of trigeminal neuralgia varied from one year to 21 years with a mean of 9 years. Five patients had disseminated sclerosis at the time of operation and one patient had acromegaly.

The diagnosis of trigeminal neuralgia was made on clinical grounds. All but three patients had plain skull radiography and four had a CT scan, none of which showed any clinical abnormality. Three patients early in the series had normal air encephalograms. All patients had previously been treated with carbamezepine in doses ranging up to $1400 \mathrm{mg}$ per day and $48 \mathrm{had}$ previously had at least one 
Table 1 Shows the procedures performed before posterior fossa surgery

\begin{tabular}{ll}
\hline Procedure & Number \\
\hline Peripheral nerve procedure (injection or avulsion) & 13 \\
Trigeminal ganglion procedure & 28 \\
$\quad$ (injection or radiofrequency) & 7 \\
Peripheral nerve and ganglion procedure & 9 \\
No other procedure & \\
\hline
\end{tabular}

procedure, such as peripheral nerve or ganglion alcohol injections, radiofrequency ganglion rhizolysis, or peripheral neurectomy (table 1). Two patients had undergone a previous middle fossa approach and partial retroganglion section of the sensory rootlets of the 5th nerve. Nine patients had no previous procedures before their posterior fossa operation. Fifty-four patients were followed up, 46 by interview with one of the authors and two by questionnaire. Six patients had died by the time of review and contact was made with their nearest living relative and local doctor in order to assess the result of the operation. Three patients could not be traced. The length of follow up varied from 8 months to 10 years with a mean of 4.5 years. Thirty-four patients were followed up for more than 5 years.

\section{Operative technique}

Patients were operated upon under general anaesthetic positioned on their side. The operative approach was through a curved retromastoid skin incision. A small suboccipital craniotomy was performed and this was extended to delineate the angle between the transverse sinus and the sigmoid sinus. After opening the dura the cerebellum was gently retracted and CSF aspirated. In many of the older patients very little retraction was needed. The 7th and 8th cranial nerves were then exposed. It was usually necessary to divide a bridging vein at this stage, often the superior petrosal vein. A thorough exploration of the 5th cranial nerve, including its junction with the brainstem, was then performed. In those cases in which no compressive lesion could be seen, either whole or part of the sensory division of the 5th nerve was sectioned, care being taken to preserve the motor root in all cases (table 2 ). In 12 cases portio intermedia fibres were seen and left intact. The 5th nerve was noted to be indented and grooved or distorted by a vascular structure in six cases, five times by the superior cerebellar artery (SCA), and once by a fusiform aneurysm of the basilar artery (table 3 ). In three of the five cases where the SCA was indenting the nerve the vessel was mobilised away from the nerve and a piece of Ivalon sponge placed between the vessel and the nerve, including one case where partial sensory root section was also performed. In the other two cases of compression by the SCA, part of the nerve was just divided. The patient in whom the compression was by a fusiform basilar artery aneurysm, the whole nerve was divided. In five patients with disseminated sclerosis a possible plaque was noted on the nerve in two, and in another two the nerve was noted to be atrophic.

In those patients who had a partial section of the sensory division of the 5 th nerve the decision concerning the amount of nerve to be sectioned and the position of the section was taken on the understanding of the knowledge of the anatomy of the nerve, ${ }^{13-15}$ although in some cases it was considered wiser that a total section be performed. Table 2 shows the percentage of the sensory division of the 5 th nerve cut in relation to the position of the pain. In those patients with first and second division pain the cranio-medial portion of the nerve was cut (that is that closest to the motor root) whereas in all others where a partial section was performed the caudo-lateral part of the nerve farthest away from the motor root was sectioned. Two patients had two sections, the first being a partial section and the second a total section

Table 2 Shows division of pain and percentage of sensory root sectioned. Two patients had two sections

\begin{tabular}{lllllr}
\hline Division of pain & \multicolumn{2}{l}{$\%$ Sectioned } & & Total \\
\cline { 2 - 5 } & $30-50$ & $55-70$ & $75-90$ & 100 & 3 \\
\hline V2 & 2 & -5 & - & 1 & 1 \\
V 31,2 & 6 & 2 & - & 2 & 4 \\
V 2,3 & $\frac{5}{4}$ & 11 & 4 & 3 & 22 \\
V $1,2,3$ & - & - & 1 & 12 & 13 \\
\hline
\end{tabular}

Table 3 Shows the procedure performed and results in patients with a vascular abnormality compressing the sensory division of the trigeminal nerve

\begin{tabular}{llll}
\hline Case & Abnormal vessel compressing nerve & Procedure & Result \\
\hline LP & Fusiform basilar aneurysm & $100 \%$ section & No further pain \\
IR & SCA & $65 \%$ section and MVD & No further pain \\
ES & SCA & $80 \%$ section & No further pain \\
RD & SCA & MVD & "Twinges" of pain and burning in tongue \\
CM & SCA & MVD & No further pain \\
MC & SCA & $70 \%$ section & No further pain \\
\hline
\end{tabular}

MVD Microvascular decompression. Ivalon sponge placed between vessel and nerve. 
Table 4 Shows relationship of percentage of sensory root sectioned to recurrence of trigeminal neuralgia

\begin{tabular}{llllll}
\hline Result & \multicolumn{2}{l}{$\%$} & & Total \\
\cline { 2 - 5 } & $30-50$ & $55-70$ & $75-90$ & 100 & \\
\hline No further pain & 10 & 15 & 4 & 19 & 48 \\
Minor twinges of trigeminal neuralgia & 1 & 3 & $-1^{*}$ & - & 4 \\
Moderate/severe trigeminal neuralgia & $1^{*}$ & - & - & 2 \\
\hline
\end{tabular}

* Patients had further total sensory root section resulting in no further trigeminal neuralgia in both but anaesthesia dolorosa in one.

Table 5 Shows results in 34 patients followed up for more than 5 years related to procedure performed

\begin{tabular}{|c|c|c|c|c|c|c|}
\hline \multirow[t]{2}{*}{ Result } & \multirow[t]{2}{*}{$M V D$} & \multicolumn{4}{|c|}{$\%$ Sectioned } & \multirow{2}{*}{$\begin{array}{l}\text { Total } \\
\text { procedures }\end{array}$} \\
\hline & & $30-50$ & $55-70$ & 75-90 & 100 & \\
\hline $\begin{array}{l}\text { No further trigeminal neuralgia } \\
\text { Minor twinges of trigeminal neuralgia } \\
\text { Moderate/severe trigeminal neuralgia }\end{array}$ & $\begin{array}{l}1 \\
1 \\
\end{array}$ & $\begin{array}{l}5 \\
1 \\
1 *\end{array}$ & $\begin{array}{l}9 \\
1 \\
-\end{array}$ & $\frac{1}{1^{*}}$ & $\frac{15}{-}$ & $\begin{array}{r}31 \\
3 \\
2\end{array}$ \\
\hline
\end{tabular}

* Patients had further total sensory root section resulting in no further trigeminal neuralgia in both but anaesthesia dolorosa in one.

MVD Microvascular decompression.

in both cases. Eleven of the 12 cases of 3 rd division pain had a section of less than $70 \%$ of the sensory root and the one patient who had a recurrence of pain following partial section then had a total division of the nerve. Fifteen of the 22 patients with 2 nd and 3 rd division pain had less than $70 \%$ of the sensory nerve cut. Of the three patients who had a total section one had multiple sclerosis and it was considered advisable to cut the nerve, one had a fusiform basilar artery aneurysm tightly compressing the whole nerve and the other patient had a previous ganglion injection resulting in 1st and 2 nd division anaesthesia anyway.

\section{Results}

All patients had left hospital by the tenth postoperative day and there was no mortality or serious morbidity associated with the operation. The results for the relief of trigeminal neuralgia are as shown in Table 4.

Forty-eight root section procedures resulted in no further trigeminal neuralgia. Thirty-four patients have been followed for more than 5 years and 29 of these had no further tic douloureux (table 5). Two of the 52 patients who had a root section $(3 \cdot 8 \%)$ had a relapse of their trigeminal neuralgia following operation; one of these patients developed further trigeminal neuralgia 4 years after a partial section and has had no further pain following a complete division of the nerve. Another patient who had a relapse of pain after 7 years had a further total section and has had no further trigeminal neuralgia but unfortunately has anaesthesia dolorosa. Of the four patients who had minor twinges of trigeminal neuralgia returning after operation, all of these occurred in areas which were not numb and none of the patients was bothered by this pain. One patient had definite anaesthesia dolorosa although a further four patients complained of "burning" or "scalding" feeling on the side of their face (table 6). This includes the one patient who had a microvascular decompression and has severe burning in his tongue. A number of patients admitted to minor "crawling" sensations beneath the skin of their face when directly questioned but considered it to be no problem. Aside from the recurrence of pain and painful dysaesthesia there has been no major morbidity associated with the operation. There were no serious corneal problems although four patients required a temporary tarsorrhaphy and one patient, previously blind, had a permanent tarsorrhaphy (table 7). One patient had probable temporary CSF otorrhoea and one a unilateral mild nerve deafness.

Table 6 Shows relationship of painful dysaesthesia to percentage of sensory division of trigeminal nerve sectioned

\begin{tabular}{|c|c|c|c|}
\hline & \multicolumn{3}{|l|}{ Procedures } \\
\hline & Partial section & Complete section & $M V D$ \\
\hline $\begin{array}{l}\text { Moderate discomfort } \\
\text { Severe discomfort }\end{array}$ & 1 & $\begin{array}{l}2 \\
1\end{array}$ & $\overline{1}$ \\
\hline
\end{tabular}

MVD Microvascular decompression.

Table 7 Shows relation of percentage of sensory nerve sectioned and occurrence of corneal problems (i.e. reddening of the eye sufficient to refer back to the Department)

\begin{tabular}{ll}
\hline$\%$ Sectioned & Number of patients \\
\hline $30-50$ & - \\
$55-70$ & 1 \\
$75-90$ & 3 \\
100 & 7 \\
Total & \\
\hline
\end{tabular}


One patient admitted to having mildly impaired balance since the operation. There was no postoperative facial weakness.

The area of numbness was carefully mapped out and the corneal reflex tested on the 46 patients who were personally examined. In those patients who had a partial section of the nerve the corneal reflex was intact in all but three patients and light touch in the first division of the trigeminal nerve was found normal in all but one. In those three patients with a partial section but no corneal reflex all had a previous ganglion injection, and an absent corneal reflex had been noted prior to posterior fossa surgery. All patients with a total section had an absent corneal reflex, even in those five patients in whom a portio intermedia was seen at surgery and left intact. One patient with an $80 \%$ sensory root section and another with a $90 \%$ section both had corneal reflexes present despite a fairly marked sensory loss extending into the 1st division of the 5th nerve.

All patients with 3 rd and/or 2nd division trigeminal neuralgia who underwent a $60 \%$ or less root section had impaired sensation in the 3rd division of the 5th nerve and the 2 nd division extending up to the lower eye lid. In only one patient did it extend above the lower eye lid and that patient had a previous ganglion alcohol injection and absent sensation in the 1st division had been noted pre-operatively. Seven of the nine patients who had a $2 / 3$ rds section and were examined had a sensory impairment to pin prick in the 1st division area although light touch sensation was present in all and their corneal reflex was intact. The other two patients had sensation impaired only below the lower eye lid. In the four patients examined who had a partial section of greater than $2 / 3$ rds of the sensory root there was impaired sensation in the 1st division region although the corneal reflex was still intact in three of them together with a relative sparing of light touch.

Six patients had a vascular abnormality associated with the 5 th nerve. (The procedure performed has been described and the results are shown in table 3.) The only recurrence of pain in this group has been in one patient (RD) who had a microvascular decompression. Twinges of trigeminal pain recurred 4 years after his operation and were later followed by a burning and scalding feeling in his tongue.

\section{Discussion}

The aetiology and treatment of trigeminal neuralgia is still controversial. The relationship between compressive lesions in the posterior fossa in trigeminal neuralgia was first decribed by Dandy ${ }^{16}$ and later by Gardner. ${ }^{17}$ Although using microneurosurgical techniques Jannetta ${ }^{51-21}$ and other workers ${ }^{681022}$ have shown a high incidence of cranial nerve root compression by vascular structures in both trigeminal neuralgia and hemifacial spasm, these findings have not been universal ${ }^{11}{ }^{12} 23$ and it might be that the part played by microvascular decompression in the aetiology of trigeminal neuralgia is less important than is commonly supposed. Although in a large percentage of cases in this series a vascular structure was seen close to the trigeminal nerve, a definite vascular compressive lesion was seen in only approximately $11 \%$ of cases. As in the aetiology of hemifacial spasm, the problem remains that there might be varying interpretations of what exactly constitutes a pathologically placed vessel. ${ }^{24} \mathrm{We}$ considered that a vessel was abnormally related to the trigeminal nerve only if it could be seen to be intending and grooving or distorting the nerve. The incidence of findings of vascular abnormality did not increase later in the series suggesting that the low rate could not be accounted for by the inexperience of the surgeon.

In this series partial section of the trigeminal root has a $5.7 \%$ recurrence rate overall or a $11.8 \%$ recurrence at 5 years, figures which compare favourably with other methods of treating trigeminal neuralgia. A major consideration in treating patients with trigeminal neuralgia is that many patients find the threat of further pain too unpleasant to live with and they therefore eventually ask for a procedure most likely to cure them. Others who are elderly may not tolerate a second major operation should the pain recur. We feel that in those patients who have a pain recurrence following a more peripheral procedure and who require posterior fossa operation, the best chance for obtaining permanent pain relief is with either a partial or total section of the posterior root.

Dandy, ${ }^{4}$ a pioneer of this approach whose technique and results had no rival, found that section of the posterior root produced freedom from pain in 246 of 250 patients. In this series 50 of 52 patients $(96 \%)$ had no recurrence of significant trigeminal neuralgia with a mean follow up of 4.5 years, and of 34 patients followed for 5 years two had a recurrence of tic douloureux. In Jannetta's series ${ }^{21}{ }^{25}$ the short term recurrence of pain using microvascular decomposition is certainly low, $11 \%$ at one year but reduced to $7.5 \%$ by a second operation and Apfelbaum, ${ }^{6}$ using microvascular decompression operation, reported a $5 \%$ recurrence of severe pain at 3 years. Burchiel, ${ }^{7}$ however, reported a $17 \%$ recurrence at 25 months following microvascular decompression where an arterial compressive loop was noted.

One of the most disturbing side effects of central trigeminal sensory root sections or radiofrequency lesions of the trigeminal ganglion nerve is that of 
anaesthesia dolorosa. In this series of 52 patients with sensory root sections $7 \cdot 7 \%$ of patients had some painful dysaesthesia but it was sufficiently severe in only one to warrant the label "anaesthesia delorosa". One further patient with a severe painful dysaesthesia did not have section of the nerve but rather had a microvascular decompression using Ivalon sponge.

Microvascular decompression has the advantage that it generally produces no sensory impairment in the face. The areas of sensory loss to pain in this series corresponded to the appropriate part of the sensory root cut. After partial section useful light touch is retained even in the analgesic areas and it does seem there is some relative sparing of this modality. Although some patients admitted to biting their tongues and having minor problems with dentures and occasionally mouth ulcers for a short time after the operation, virtually all rapidly became used to this type of inconvenience. All patients with no further significant trigeminal neuralgia were pleased to have had an operation which despite numbing the face to some degree had cured the pain. No patient developed keratitis and in all those patients who had previously had an intact corneal response this was maintained following their partial section.

The anatomy of the trigeminal root is all important with this approach. Dandy ${ }^{26}$ originally suggested that section of the posterior half of the sensory root cured pain in any division with no loss of touch sensation and therefore thought that the fibres subserving pain sensation occurred in the posterior part of the sensory root with variations which accounted for recurrences after the division of this portion of this nerve. Whilst our results would partially agree with this theory they would be more in keeping with the theory of Frazier, ${ }^{21327}$ that the anatomical organisation of the trigeminal sensory root is approximately representative of the topographical divisions for all sensory modalities as represented on the face, rather than the accumulation of the fibres responsible for pain within the posterior half of the root. However, there is a variable relationship between the placement of these topographical divisions in the root near the brain stem due to the variable degree of rotation of the nerve root as it enters the brain stem. ${ }^{14} 15$ This can be appreciated if one realises the 1st division fibres lie adjacent to the motor root and the 3rd division fibres lie on the side opposite to the motor root irrespective of how much rotation occurs (figure).

There is no overall agreement on the correct operative approach for the treatment of trigeminal neuralgia. Jannetta ${ }^{25}$ compared the results of microvascular decompression with those of radiofrequency rhizotomy and considered that in view of his published low recurrence rate and

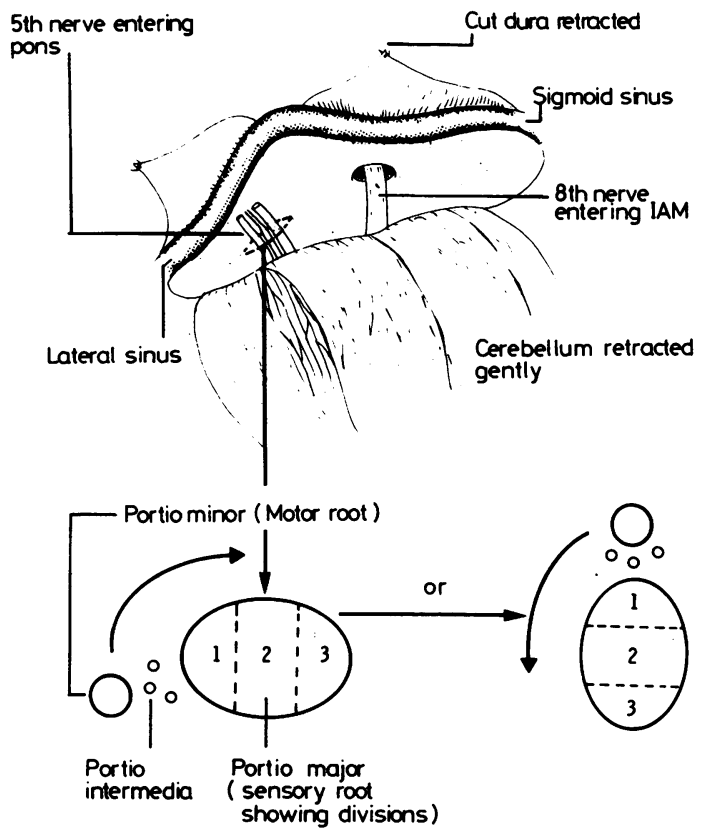

Figure Diagram to illustrate the varying degree of rotation of the trigeminal roots as they enter the brain stem. It should be noted that the majority of sensory fibres from the 3rd division of the trigeminal nerve maintain a relation to the part of the sensory root most removed from the motor root or roots.

freedom from side effects of dysaesthesia, microvascular decompression is more appropriate for patients under the age of 70 . This is supported by Loftman $^{28}$ but Guidetti ${ }^{29}$ feels that radiofrequency rhizotomy should be the treatment for younger patients followed by an extradural approach to the trigeminal ganglion for recurrences since posterior fossa approach was potentially hazardous. This is not our experience. Apfelbaum ${ }^{6}$ considers microvascular decompression preferable to percutaneous neurolysis in terms of a low incidence of recurrence and freedom from facial anaesthesia, although Burchiel's results were less convincing. ${ }^{7}$ Nugent $^{30}$ finding a $21 \%$ recurrence at 8 years in 800 patients undergoing radiofrequency rhizotomy suggested that this should be the treatment of choice initially since he estimated that the chances of recurrence from microvascular decompression to be $17 \%$ with a $2 \%$ risk of significant morbidity and mortality. He thought that a further $24 \%$ might well need a section of the posterior root with production of numbness thereafter.

Clearly the most appropriate procedure is that which carries the smallest risk for the patient but which will bring about a satisfactory pain relief with minimal side effect. This obviously varies from 
patient to patient and from surgeon to surgeon. Generally we advise a temporary method of numbing the face to allow the patient to appreciate just what the altered sensation of the face is like. Thus we use infraorbital nerve avulsion, radiofrequency rhizotomy or retrogasserian glycerol injection accepting that for all these there is a high recurrence rate: However, some patients find the threat of further pain in the future unacceptable and for these patients a posterior fossa exploration is particularly indicated. If there is clear evidence of vascular compression then a microvascular decompression should be performed, However, as we have found the incidence of vascular compression to be low then partial or total root section has been performed in our series-the degree of section depending on the area of trigeminal neuralgia.

The early good results associated with microvascular decompression might be related to the manipulation of the nerve as was seen after the "compression-decompression" operation of Taarnhoj. ${ }^{31}{ }^{32}$ For the last 6 years we have successfully treated hemifacial spasm by wrapping the facial nerve with Ivalon sponge so as to produce circumferential fibrosis. We feel it logical to wrap the trigeminal nerve in the same way with the same aim for trigeminal neuralgia. We have carried out this procedure recently with relief of trigeminal neuralgia and minimum impairment of facial sensation.

We report our experiences not only to record our failure to be convinced by vascular compression as a cause for the majority of our patients' pain but also to point out that the posterior fossa approach using microsurgical techniques is extremely safe as well as effective. Furthermore it is possible with this approach to carry out a partial section of the trigeminal root to spare the upper part of the face and cornea when the pain is confined to the 2 nd and 3 rd divisions of the trigeminal nerve-such a section also preserving some sensation of touch even in the analgesic zone. Total section of the sensory root, even with preservation of the portio intermedia and minor fibres invariably produces complete analgesia and anaesthesia. In this respect we differ from Dandy who ascribed preservation of sensation to sparing of the portio intermedia. Further significant advantages of the posterior approach are the ease with which motor root and facial nerve can be spared, the confidence with which one can completely sever the main sensory root, should that be indicated, and finally the ease with which positive pathology can be identified and treated.

\footnotetext{
References

' Horsley V. An address on the surgical treatment of trigeminal neuralgia. Practitioner 1900;65:251-3.
}

2 Frazier $\mathrm{CH}$. Subtotal resection of sensory root for relief of major trigeminal neuralgia. Arch Neurol Psychiatry 1925;13:378-84.

${ }^{3}$ Spiller WG, Frazier $\mathrm{CH}$. The division of the sensory root of the trigeminus for the relief of tic douloureux. University Pennsylvania Medical Bulletin 1901;14:341.

${ }^{4}$ Dandy, WE. The treatment of trigeminal neuralgia by the cerebellar root. Ann Surg 1932;96:787-95.

5 Jannetta PJ. Microsurgical approaches to the trigeminal nerve for tic douloureux. Progress of Neurological Surgery 1976;7:180-200.

- Apfelbaum RI. A comparison of percutaneous radiofrequency, trigeminal neurolysis and microvascular decompression of the trigeminal nerve for treatment of tic douloureux. Neurosurgery 1977;1:16-21.

${ }^{7}$ Burchiel KM, Steege TD, Howe JF, Loeser JB. Comparison of percutaneous radiofrequency gangliolysis and microvascular decompression for the surgical management of tic douloureux. Neurosurgery 1981;9;2:111-9.

${ }^{8}$ Petty PG, Southly R. Vascular decompression of the lower cranial nerves. Observations using the microscope with particular reference to trigeminal neuralgia. Aust NZ J Surg 1977;47:314-20.

9 Weidmann MJ. Trigeminal neuralgia. Surgical treatment by microvascular decompression of the trigeminal root. Med J Aust 1979;2:628-30.

${ }^{10}$ Wilson CB, Yorke C, Prioleau G. Microsurgical vascular decompression for trigeminal neuralgia and hemifacial spasm. West J Med 1980;132:481-4.

"Fabinyi GCA, Adams CBT. Hemifacial spasm: treatment by posterior fossa surgery. J Neurol Neurosurg Psychiatry 1978;41:829-33.

${ }^{12}$ Kaye AH, Adams CBT. Hemifacial spasm. A long term follow up of patients treated with posterior fossa surgery. J Neurol Neurosurg Psychiatry 1981;44:11003.

${ }^{13}$ Poulos DA. Functional and anatomical localization in the trigeminal root. In support of Frazier. Morley TP, ed. Current Controversies in Neurosurgery. Philadelphia: WB Saunders, 1976:539-45.

${ }^{14}$ Gudmundsson K, Rhoton AL, Rushton JG. Detailed anatomy of the intracranial portion of the trigeminal nerve. J Neurosurg 1971;35:592-600.

15 Pelletier VA, Poulos DA, Lende RA. Functional localization in the trigeminal root. $J$ Neurosurg 1974;40:504-13.

${ }^{16}$ Dandy WE. Concerning the cause of trigeminal neuralgia. Am J Surg 1934;24:447-55.

${ }^{17}$ Gardner WJ. Concerning the mechanisms of trigeminal neuralgia and hemifacial spasm. $J$ Neurosurg 1962;19:947-58.

18 Jannetta PJ. Arterial compression of the trigeminal nerve at the pons in patients with trigeminal neuralgia. $J$ Neurosurg 1967;26:159-62.

19 Jannetta PJ. Treatment of trigeminal neuralgia by suboccipital and transtentorial cranial operations. Clin Neurosurg 1976;24:538-49.

${ }^{20}$ Jannetta PJ, Abassy M, Maroon JC, Ramos FM, Albir MS. Aetiology and definitive microsurgical treatment of hemifacial spasm. J Neurosurg 1977;47:321-8. 
${ }^{21}$ Jannetta PJ, Zorub DS. Microvascular decompression for trigeminal neuralgia. Burcheit WA, Truex RC Jnr, eds.Surgery of the posterior fossa. New York: Raven Press 1979:143-54.

${ }^{22}$ Gardner WJ, Savar GA. Hemifacial spasm-a reversible pathophysiological state. J Neurosurg 1962;19:240-7.

${ }^{23}$ Tarlov E. Percutaneous and open microneurosurgical techniques for relief of refractory tic douloureux. Surg Clin North Am 1980;60;2:593-607.

${ }^{24}$ Morley TP. Introduction to article by Jannetta PJ. Morley TP, ed. Current Controversies in Neurosurgery. Philadelphia: Saunders WB, 1976.

${ }^{25}$ Jannetta PJ. Treatment of trigeminal neuralgia. Viewpoint. Neurosurgery 4;1:93-4.

${ }^{26}$ Dandy WE. An operation for the cure of tic douloureux. Partial section of the sensory root of the pons. Arch
Surg 1929;18:687-93.

${ }^{27}$ Frazier $\mathrm{CH}$, Whitehead E. The morphology of the gasserian ganglion. Brain 1925;48:458-75.

${ }^{28}$ Loftman BA. Trigeminal neuralgia. Am Surg 1979;45:(10):665-9.

${ }^{29}$ Guidetti B, Fraiolo B, Refice GM. Modern trends in the surgical treatment of trigeminal neuralgia. J Maxillofac Surg 1979;7:315-9.

${ }^{30}$ Nugent RG. Comment on article by Burchiel KJ et al. Neurosurgery 1981;9;2:118-9.

${ }^{31}$ Taarnhoj P. Decompression of the trigeminal root and the posterior part of the ganglion as a treatment in trigeminal neuralgia; preliminary communication. $J$ Neurosurg 1952;9:288-90.

${ }^{32}$ Taarnhoj P. Decompression of the trigeminal root. $J$ Neurosurg 1954;11:299-305. 